\title{
DIFERENTES ARRANJOS PARA ESTUDO DE BORDADURA LATERAL EM PARCELAS EXPERIMENTAIS DE MILHO-VERDE ${ }^{1}$
}

\author{
SUELI MARTINS DE FREITAS ALVES ${ }^{2}$, JOSÉ CARLOS SERAPHIN ${ }^{3}$, \\ ÁLVARO ELEUTÉRIO DA SILVA ${ }^{4}$ e FRANCISCO JOSÉ P. ZIMMERMANN ${ }^{5}$
}

\begin{abstract}
RESUMO - Visando ao estudo do uso de bordadura lateral em experimentos de avaliação de genótipos de milho-verde, testaram-se quatro arranjos para pré-especificar a disposição das linhas na parcela experimental básica. No arranjo 1, o estudo foi feito de forma que os efeitos convergissem para o centro da parcela. No arranjo 2, têm-se informações do efeito de bordadura de cada lado da parcela. No arranjo 3, as informações são obtidas em cada linha da parcela, e no arranjo 4, têm-se informações das linhas de cada lado da parcela em relação à parte central. Os dados utilizados foram provenientes de um ensaio de avaliação de 20 genótipos de milho-verde, coletados separadamente em cada linha da parcela, permitindo-se, desta maneira, utilizar o esquema de parcelas subdivididas. Os resultados obtidos possibilitam recomendar os arranjos 1 ou 2 para o estudo de bordadura lateral. Com relação à cultura de milho-verde, verificou-se que há necessidade de duas fileiras de bordadura lateral de cada lado da parcela.
\end{abstract}

Termos para indexação: Zea mays, cultivares, genótipos, disposição de linhas, métodos, cultivo de grãos.

\section{SIDE BORDER ARRANGEMENT IN EXPERIMENTAL PLOTS OF GREEN CORN}

\begin{abstract}
In order to study the use of side borders in green corn genotypes evaluation experiments, four arrangements were tested to pre-specify the number of rows in the basic experimental plot. In the arrange 1 the side border study was organized in a way that the results should converge to the center of the plot; in the arrange 2, information was obtained about each side border of the plot; for arrange 3, the information obtained quantifies this effect for each row of the plot; and arrange 4 has provided information about the effect of side related to the central portion of the plot. For this study, data from a 20 corn genotypes evaluation trial for in natura consumption were utilized. Data were separately annotated for each row of the plot, thus permitting utilization of the split-plot scheme. In similar condition arrange 1 or 2 may be recommended for lateral border study, and for corn in natura consumption a two-row side border is required for each side of the plot.
\end{abstract}

Index terms: Zea mays, cultivars, genotypes, band placement, evaluation, methods, grain crops.

\section{INTRODUÇÃO}

O efeito de competição entre plantas de parcelas adjacentes pode fazer com que o desempenho de

${ }^{1}$ Aceito para publicação em 10 de janeiro de 2000.

${ }^{2}$ Eng. Agrôn., M.Sc., Instituto de Matemática e Estatística (IME), Universidade Federal de Goiás (UFG), Caixa Postal 131, CEP 74001-970 Goiânia, GO. E-mail: sueli@mat.ufg.br

${ }^{3}$ Eng. Agrôn., Ph.D., IME, UFG. E-mail: seraphin@mat.ufg.br

${ }^{4}$ Eng. Agrôn., Ph.D., Agência Rural, Diretoria de Pesquisa, Caixa Postal 331, CEP 74610-060 Goiânia, GO.

${ }^{5}$ Eng. Agrôn., Ph.D., Embrapa-Centro Nacional de Pesquisa de Arroz e Feijão (CNPAF), Caixa Postal 179, CEP 75375-000 Santo Antônio de Goiás, GO.

E-mail: zimmermann@cnpaf.embrapa.br determinados genótipos seja alterado, afetando a comparação entre eles. Este efeito afeta a precisão experimental, e pode variar entre espécies, cultivares, experimentos, e até mesmo de acordo com os caracteres avaliados (Gomez \& Gomez, 1984; Silva et al., 1991).

Para minimizar a influência mútua entre parcelas adjacentes, pode-se utilizar fileiras externas nas parcelas, ou seja, bordadura lateral, as quais não são aproveitadas para a obtenção dos dados experimentais. Estas fileiras aumentarão o tamanho da parcela, e podem acarretar um aumento da heterogeneidade entre parcelas, e, por conseguinte, um maior erro experimental (Valentini et al.,1988). Embora essas fileiras muitas vezes sejam necessárias, seu uso siste- 
mático e indiscriminado e sem nenhum estudo prévio deve ser evitado.

Para verificar se realmente se justifica o uso de fileiras de bordadura, um método comumente empregado é o proposto por Gomez \& Gomez (1984), que consiste em impor um delineamento em parcelas subdivididas sobre o delineamento original do experimento, onde as subparcelas têm uma disposição préespecificada.

Nas pesquisas de competição de cultivares, realizadas em diversas culturas com o objetivo de avaliar a necessidade de fileiras de bordadura lateral, verifica-se que quando há efeito de competição, o número de fileiras a serem descartadas de cada lado da parcela varia conforme a cultura. Em culturas como milho para grão, em que o espaçamento entre linhas é grande, geralmente uma linha de bordadura de cada lado pode ser suficiente (Gomez \& Gomez, 1984). Para culturas como arroz, em que o espaçamento entre linhas é menor, Gomez (1972) e Zimmermann (1980) recomendam o uso de duas fileiras de bordadura lateral, como forma de aumentar a precisão experimental. Porém, quando não é detectada influência significativa das bordaduras sobre os resultados obtidos nas fileiras centrais, estas tornam-se desnecessárias, como foi verificado na cultura de feijão por Arruda (1959), Vieira (1964) e Valentini et al. (1988). Embora o tema bordadura já tenha sido abordado em várias culturas, não se tem informação sobre milho-verde, cultura esta que apresenta uma importância econômica e social bastante significativa.

O objetivo deste trabalho foi avaliar o uso de bordadura lateral em experimentos de avaliação de genótipos de milho-verde.

\section{MATERIAL E MÉTODOS}

Os dados utilizados neste trabalho foram provenientes de um ensaio de avaliação de 20 genótipos de milho-verde: AGROMEN 2012; XL 660; XL 655; XL 604; C 742; C 505; AG 519; AG 951; AG 4591; AG 7391; PIONEER 3232; DINA 170; AG 1051; EMGOPA 501; AG 603; BR 112; BR 106; BR 126; CMS 39; CMS 50 X 28. Este ensaio foi instalado no mês de maio de 1995, na estação experimental da Embrapa-Centro Nacional de Pesquisa de Arroz e Feijão, localizada no município de Santo Antônio de Goiás, GO.
O delineamento utilizado foi o de blocos completos casualizados, com quatro repetições. As parcelas experimentais foram compostas de seis linhas de $5 \mathrm{~m}$ de comprimento, com espaços, entre si, de $0,90 \mathrm{~m}$, com uma densidade de cinco plantas por metro linear.

Por ocasião da colheita, todas as fileiras foram avaliadas quanto à altura da planta em $\mathrm{cm}$ (do nível do solo à folha bandeira; média de 10 plantas competitivas) e peso de espiga com palha ( $\mathrm{kg} / \mathrm{ha}$ ).

Para medir o efeito de bordadura lateral, utilizou-se o método proposto por Gomez \& Gomez (1984). A parcela experimental básica é subdividida em pares das linhas externas, pares das linhas adjacentes às linhas externas e assim sucessivamente até as linhas centrais. Esta disposição das linhas corresponde ao primeiro arranjo proposto. Outros três arranjos foram propostos para pré-especificar a disposição das linhas na parcela experimental básica.

No arranjo 1, as parcelas foram subdivididas em três posições: $\mathrm{P}_{1}$ (linha 1 e linha 6), $\mathrm{P}_{2}$ (linha 2 e linha 5) e $\mathrm{P}_{3}$ (linha 3 e linha 4). No arranjo 2, as parcelas também foram subdivididas em três posições: $\mathrm{P}_{1}$ (linha 1 e linha 2), $\mathrm{P}_{2}$ (linha 5 e linha 6) e $\mathrm{P}_{3}$ (linha 3 e linha 4). No arranjo 3, as parcelas foram subdivididas em seis posições, uma para cada linha e, no arranjo 4, elas foram subdivididas em cinco posições: $P_{1}$ (linha 1), $P_{2}$ (linha 2), $P_{3}$ (linha 3 e linha 4), $\mathrm{P}_{4}$ (linha 5) e $\mathrm{P}_{5}$ (linha 6). Após a obtenção de cada arranjo, com relação a cada caráter estudado, foram efetuadas análises de variância considerando o modelo matemático do delineamento em blocos completos casualizados, com parcelas subdivididas.

\section{RESULTADOS E DISCUSSÃO}

A confiabilidade dos resultados obtidos em um experimento depende do controle do erro experimental; assim, a correta interpretação desses experimentos depende da precisão com que foram planejados, instalados e conduzidos. Como indicador da precisão experimental pode-se utilizar o coeficiente de variação. Na Tabela 1, pode-se observar que as estimativas do coeficiente de variação referente à variável altura das plantas (ALTPL) foram relativamente baixas em comparação com as encontradas na variável peso das espigas com palha (PEP), provavelmente pelo fato de a influência dos efeitos ambientais ser mais intensa sobre este último caráter. Segundo a classificação dos coeficientes de variação na cultura de milho proposta por Scapim et al. (1995), as estimativas obtidas na variável ALTPL encontram-se altas nas parcelas, e médias nas subparcelas, e na variável 
PEP variam de médio a alto nas parcelas e médio nas subparcelas. Verifica-se, também, que os quadrados médios residuais, e, conseqüentemente, os coeficientes de variação nos dois primeiros arranjos foram menores, conferindo, assim, aos arranjos 1 e 2, maior precisão em comparação com os arranjos 3 e 4 .

Na Tabela 1, verifica-se, ainda, que, no que diz respeito à variável peso de espigas com palha, houve, em todos os arranjos, um efeito significativo de posição; também foi detectado, nos arranjos 1, 2 e 3, um efeito significativo na interação genótipo x posição. Observa-se, também, que os quadrados médios do efeito de posição são consideravelmente maiores do que os quadrados médios da interação, o que confere ao efeito de posição uma magnitude maior para a estatística $F$.

O estudo do desdobramento de posição dentro de cada genótipo é apresentado nas Tabelas 2, 3, 4 e 5 , nas quais pode-se observar que o efeito de competição variou entre os genótipos. Na Tabela 2, arranjo 1, constata-se que na variável ALTPL, 17 dos 20 genótipos estudados não apresentaram diferenças significativas entre as posições, e apenas os genótipos AGROMEN 2012, DINA 170 e BR 112 apresentaram altura estatisticamente superior na posição mais central da parcela, ou seja, as posições mais externas poderiam ter sofrido influência das parcelas adjacentes. $\mathrm{O}$ efeito de bordadura, não tem sido muito freqüente em relação a esta variável, como se pode verificar em Zimmermann (1980) em arroz, Valentini et al. (1988) em feijão, e Silva et al. (1991) em milho irrigado.

No arranjo 1 (Tabela 3), arranjo 2 (Tabela 4) e arranjo 3 (Tabela 5), a variável peso de espigas com palha, apresentou diferenças significativas entre as posições em 6,4 e 5 dos 20 genótipos estudados, respectivamente. Nesses materiais foi verificado, em relação ao arranjo 1 (Tabela 3), um decréscimo na produção da posição externa variando entre $12,18 \%$ e $25,17 \%$, e na posição contígua à externa, entre $22,00 \%$ e 34,14\%, em comparação com a posição central. Em relação ao arranjo 2 (Tabela 4), o decréscimo na produção da posição central variou de $9,75 \%$ a $23,30 \%$ na posição 1 e de $17,38 \%$ a $36,00 \%$ na posição 2. No arranjo 3 (Tabela 5), este decréscimo em relação a média de $\mathrm{P} 3$ e P4 variou de $18,35 \%$ a $28,44 \%$ na posição 1 , de $13,00 \%$ a $28,25 \%$ na posição 2 , de

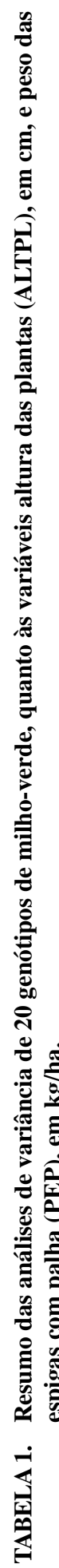


TABELA 2. Altura média das plantas de 20 genótipos de milho-verde, em cada posição $\left(\mathbf{P}_{1}, \mathbf{P}_{2}, \mathbf{P}_{3}\right)$, para o arranjo $1^{1}$.

\begin{tabular}{lccc}
\hline Genótipo & \multicolumn{3}{c}{ Altura das plantas $(\mathrm{cm})$} \\
\cline { 2 - 4 } & $\mathrm{P}_{1}$ & $\mathrm{P}_{2}$ & $\mathrm{P}_{3}$ \\
\hline AGROMEN 2012 & $161,72 \mathrm{~b}$ & $166,67 \mathrm{~b}$ & $177,70 \mathrm{a}$ \\
XL 660 & $180,07 \mathrm{a}$ & $182,27 \mathrm{a}$ & $181,82 \mathrm{a}$ \\
XL 655 & $184,85 \mathrm{a}$ & $180,15 \mathrm{a}$ & $180,65 \mathrm{a}$ \\
XL 604 & $182,90 \mathrm{a}$ & $181,37 \mathrm{a}$ & $180,95 \mathrm{a}$ \\
C 742 & $175,45 \mathrm{a}$ & $175,85 \mathrm{a}$ & $175,17 \mathrm{a}$ \\
C 505 & $187,77 \mathrm{a}$ & $194,75 \mathrm{a}$ & $183,62 \mathrm{a}$ \\
AG 519 & $180,60 \mathrm{a}$ & $177,55 \mathrm{a}$ & $182,35 \mathrm{a}$ \\
AG 951 & $192,77 \mathrm{a}$ & $193,82 \mathrm{a}$ & $192,42 \mathrm{a}$ \\
AG 4591 & $184,12 \mathrm{a}$ & $181,95 \mathrm{a}$ & $191,02 \mathrm{a}$ \\
AG 7391 & $181,07 \mathrm{a}$ & $184,67 \mathrm{a}$ & $187,20 \mathrm{a}$ \\
PIONEER 3232 & $196,12 \mathrm{a}$ & $205,32 \mathrm{a}$ & $197,65 \mathrm{a}$ \\
DINA 170 & $204,87 \mathrm{~b}$ & $218,10 \mathrm{a}$ & $215,45 \mathrm{a}$ \\
AG 1051 & $189,37 \mathrm{a}$ & $190,82 \mathrm{a}$ & $193,82 \mathrm{a}$ \\
EMGOPA 501 & $206,32 \mathrm{a}$ & $202,25 \mathrm{a}$ & $205,37 \mathrm{a}$ \\
AG 603 & $176,60 \mathrm{a}$ & $180,45 \mathrm{a}$ & $184,15 \mathrm{a}$ \\
BR 112 & $160,77 \mathrm{~b}$ & $172,97 \mathrm{a}$ & $170,57 \mathrm{a}$ \\
BR 106 & $188,40 \mathrm{a}$ & $195,23 \mathrm{a}$ & $196,57 \mathrm{a}$ \\
BR 126 & $197,35 \mathrm{a}$ & $188,12 \mathrm{a}$ & $192,20 \mathrm{a}$ \\
CMS 39 & $208,57 \mathrm{a}$ & $201,25 \mathrm{a}$ & $201,90 \mathrm{a}$ \\
CMS 50 x 28 & $187,72 \mathrm{a}$ & $179,47 \mathrm{a}$ & $182,40 \mathrm{a}$ \\
\hline
\end{tabular}

${ }^{1}$ Médias seguidas da mesma letra, na linha, não diferem estatisticamente entre si pelo teste de Scott-Knott a 5\% de probabilidade.

TABELA 3. Peso médio das espigas com palha de 20 genótipos de milho-verde, em cada posição $\left(\mathbf{P}_{1}, \mathbf{P}_{2}, \mathbf{P}_{3}\right)$, para o arranjo $1^{1}$.

\begin{tabular}{lccc}
\hline Genótipo & \multicolumn{3}{c}{ Peso das espigas (kg/ha) } \\
\cline { 2 - 4 } & $\mathrm{P}_{1}$ & $\mathrm{P}_{2}$ & $\mathrm{P}_{3}$ \\
\hline AGROMEN 2012 & $14438,89 \mathrm{a}$ & $13658,33 \mathrm{a}$ & $13961,11 \mathrm{a}$ \\
XL 660 & $14808,33 \mathrm{a}$ & $13375,00 \mathrm{a}$ & $15166,67 \mathrm{a}$ \\
XL 655 & $13330,56 \mathrm{a}$ & $12683,33 \mathrm{a}$ & $15102,78 \mathrm{a}$ \\
XL 604 & $14913,89 \mathrm{a}$ & $15233,33 \mathrm{a}$ & $15505,56 \mathrm{a}$ \\
C 742 & $15969,44 \mathrm{a}$ & $12983,33 \mathrm{~b}$ & $15488,89 \mathrm{a}$ \\
C 505 & $13738,89 \mathrm{a}$ & $13744,44 \mathrm{a}$ & $13355,56 \mathrm{a}$ \\
AG 519 & $13472,22 \mathrm{a}$ & $11727,78 \mathrm{a}$ & $14430,56 \mathrm{a}$ \\
AG 951 & $16305,56 \mathrm{~b}$ & $15788,89 \mathrm{~b}$ & $18566,67 \mathrm{a}$ \\
AG 4591 & $14975,00 \mathrm{a}$ & $15744,44 \mathrm{a}$ & $14741,67 \mathrm{a}$ \\
AG 391 & $15491,67 \mathrm{a}$ & $15255,56 \mathrm{a}$ & $16033,33 \mathrm{a}$ \\
PIONEER 3232 & $14575,00 \mathrm{a}$ & $13341,67 \mathrm{a}$ & $12466,67 \mathrm{a}$ \\
DINA 170 & $12347,22 \mathrm{~b}$ & $10866,67 \mathrm{~b}$ & $16500,00 \mathrm{a}$ \\
AG 1051 & $15222,22 \mathrm{a}$ & $14972,22 \mathrm{a}$ & $16472,22 \mathrm{a}$ \\
EMGOPA 501 & $10594,44 \mathrm{~b}$ & $10544,44 \mathrm{~b}$ & $13561,11 \mathrm{a}$ \\
AG 603 & $15813,89 \mathrm{a}$ & $14052,78 \mathrm{a}$ & $16186,11 \mathrm{a}$ \\
BR 112 & $11427,78 \mathrm{~b}$ & $13266,67 \mathrm{a}$ & $14319,44 \mathrm{a}$ \\
BR 106 & $12916,67 \mathrm{a}$ & $13119,44 \mathrm{a}$ & $12541,67 \mathrm{a}$ \\
BR 126 & $12158,33 \mathrm{~b}$ & $12525,00 \mathrm{~b}$ & $16061,11 \mathrm{a}$ \\
CMS 39 & $11875,00 \mathrm{a}$ & $12308,33 \mathrm{a}$ & $12844,44 \mathrm{a}$ \\
CMS 50 x 28 & $11305,56 \mathrm{a}$ & $10519,44 \mathrm{a}$ & $11261,11 \mathrm{a}$ \\
\hline
\end{tabular}

${ }^{1}$ Médias seguidas da mesma letra, na linha, não diferem estatisticamente pelo teste de Scott-Knott a 5\% de probabilidade.
TABELA 4. Peso médio de espigas com palha de 20 genótipos de milho-verde, em cada posição $\left(\mathbf{P}_{1}, \mathbf{P}_{2}, \mathbf{P}_{3}\right)$, para o arranjo $2^{1}$.

\begin{tabular}{lccc}
\hline Genótipo & \multicolumn{3}{c}{ Peso das espigas (kg/ha) } \\
\cline { 2 - 4 } & $\mathrm{P}_{1}$ & $\mathrm{P}_{2}$ & $\mathrm{P}_{3}$ \\
\hline AGROMEN 2012 & $14127,78 \mathrm{a}$ & $13969,44 \mathrm{a}$ & $13961,11 \mathrm{a}$ \\
XL 660 & $13752,78 \mathrm{a}$ & $14430,56 \mathrm{a}$ & $15166,67 \mathrm{a}$ \\
XL 655 & $13147,22 \mathrm{a}$ & $12866,67 \mathrm{a}$ & $15102,78 \mathrm{a}$ \\
XL 604 & $15580,56 \mathrm{a}$ & $14566,67 \mathrm{a}$ & $15505,56 \mathrm{a}$ \\
C 742 & $13541,67 \mathrm{a}$ & $15411,11 \mathrm{a}$ & $15488,89 \mathrm{a}$ \\
C 505 & $13441,67 \mathrm{a}$ & $14041,67 \mathrm{a}$ & $13355,56 \mathrm{a}$ \\
AG 519 & $13022,22 \mathrm{a}$ & $12177,78 \mathrm{a}$ & $14430,56 \mathrm{a}$ \\
AG 951 & $16755,56 \mathrm{~b}$ & $15338,89 \mathrm{~b}$ & $18566,67 \mathrm{a}$ \\
AG 4591 & $16075,00 \mathrm{a}$ & $14644,44 \mathrm{a}$ & $14741,67 \mathrm{a}$ \\
AG 7391 & $15766,67 \mathrm{a}$ & $14980,56 \mathrm{a}$ & $16033,33 \mathrm{a}$ \\
PIONEER 3232 & $13938,89 \mathrm{a}$ & $13977,78 \mathrm{a}$ & $12466,67 \mathrm{a}$ \\
DINA 170 & $12655,56 \mathrm{~b}$ & $10558,33 \mathrm{~b}$ & $16500,00 \mathrm{a}$ \\
AG 1051 & $15186,11 \mathrm{a}$ & $15008,33 \mathrm{a}$ & $16472,22 \mathrm{a}$ \\
EMGOPA 501 & $10897,22 \mathrm{~b}$ & $10241,67 \mathrm{~b}$ & $13561,11 \mathrm{a}$ \\
AG 603 & $15555,56 \mathrm{a}$ & $14311,11 \mathrm{a}$ & $16186,11 \mathrm{a}$ \\
BR 112 & $12636,11 \mathrm{a}$ & $12058,33 \mathrm{a}$ & $14319,44 \mathrm{a}$ \\
BR 106 & $12755,56 \mathrm{a}$ & $13280,56 \mathrm{a}$ & $12541,67 \mathrm{a}$ \\
BR 126 & $12758,33 \mathrm{~b}$ & $11925,00 \mathrm{~b}$ & $16061,11 \mathrm{a}$ \\
CMS 39 & $12238,89 \mathrm{a}$ & $11944,44 \mathrm{a}$ & $12844,44 \mathrm{a}$ \\
CMS 50 x 28 & $10452,78 \mathrm{a}$ & $11372,22 \mathrm{a}$ & $11261,11 \mathrm{a}$ \\
\hline
\end{tabular}

${ }_{1}^{1}$ Médias seguidas da mesma letra, na linha, não diferem estatisticamente pelo teste de Scott-Knott a 5\% de probabilidade.

$22,90 \%$ a $40,00 \%$ na posição 5 , e de $28,61 \%$ a $31,99 \%$ na posição 6 . A interpretação desses resultados deve ser feita de forma diferenciada, uma vez que no arranjo 1 o efeito da bordadura é estudado de forma que os resultados convirjam para o centro da parcela; no arranjo 2, têm-se informações do efeito que ocorre de cada lado da parcela e no arranjo 3, as informações são obtidas individualmente em cada linha. Contudo, esses resultados mostram que as posições externas poderiam ter sofrido influência das parcelas adjacentes, caracterizando, assim, a possibilidade de esses materiais terem baixa capacidade de competição intergenotípica.

Pelas Tabelas 3 e 4, percebe-se também que a forma como foram arranjadas as linhas interferiu nos resultados obtidos nos genótipos C742 e BR 112, que, em relação ao arranjo 1 (Tabela 3), apresentaram diferenças estatísticas entre as posições, enquanto no arranjo 2 (Tabela 4) verifica-se que as diferenças entre as posições não foram significativas, sugerindo, assim, que não houve efeito de competição entre esses materiais, de cada lado da parcela. A partir dessas observações, levanta-se a hipótese 
TABELA 5. Peso médio de espigas com palha de 20 genótipos de milho-verde, em cada posição $\left(P_{1}, P_{2}, P_{3}, P_{4}\right.$, $\left.P_{5}, P_{6}\right)$, para o arranjo $3^{1}$.

\begin{tabular}{lrrrrrr}
\hline Genótipo & \multicolumn{7}{c}{ Peso das espigas $(\mathrm{kg} / \mathrm{ha})$} \\
\cline { 2 - 7 } & $\mathrm{P}_{1}$ & \multicolumn{1}{c}{$\mathrm{P}_{2}$} & $\mathrm{P}_{3}$ & \multicolumn{1}{c}{$\mathrm{P}_{4}$} & $\mathrm{P}_{5}$ & $\mathrm{P}_{6}$ \\
\hline AGROMEN 2012 & $13772,22 \mathrm{a}$ & $14483,33 \mathrm{a}$ & $14555,56 \mathrm{a}$ & $13366,67 \mathrm{a}$ & $12833,33 \mathrm{a}$ & $15105,56 \mathrm{a}$ \\
XL 660 & $14177,78 \mathrm{a}$ & $13327,78 \mathrm{a}$ & $14255,56 \mathrm{a}$ & $16077,78 \mathrm{a}$ & $13422,22 \mathrm{a}$ & $15438,89 \mathrm{a}$ \\
XL 655 & $13655,56 \mathrm{a}$ & $12638,89 \mathrm{a}$ & $15194,44 \mathrm{a}$ & $15011,11 \mathrm{a}$ & $12727,78 \mathrm{a}$ & $13005,56 \mathrm{a}$ \\
XL 604 & $16938,89 \mathrm{a}$ & $14222,22 \mathrm{a}$ & $16277,78 \mathrm{a}$ & $14733,33 \mathrm{a}$ & $16244,44 \mathrm{a}$ & $12888,89 \mathrm{a}$ \\
C 742 & $15188,89 \mathrm{a}$ & $11894,44 \mathrm{~b}$ & $14672,22 \mathrm{a}$ & $16305,56 \mathrm{a}$ & $14072,22 \mathrm{a}$ & $16750,00 \mathrm{a}$ \\
C 505 & $13522,22 \mathrm{a}$ & $13361,11 \mathrm{a}$ & $13544,44 \mathrm{a}$ & $13166,67 \mathrm{a}$ & $14127,78 \mathrm{a}$ & $13955,56 \mathrm{a}$ \\
AG 519 & $14572,22 \mathrm{a}$ & $11472,22 \mathrm{a}$ & $14738,89 \mathrm{a}$ & $14122,22 \mathrm{a}$ & $11983,33 \mathrm{a}$ & $12372,22 \mathrm{a}$ \\
AG 951 & $17366,67 \mathrm{a}$ & $16144,44 \mathrm{~b}$ & $18100,00 \mathrm{a}$ & $19033,33 \mathrm{a}$ & $15433,33 \mathrm{~b}$ & $15244,44 \mathrm{~b}$ \\
AG 4591 & $16561,11 \mathrm{a}$ & $15588,89 \mathrm{a}$ & $14850,00 \mathrm{a}$ & $14633,33 \mathrm{a}$ & $15900,00 \mathrm{a}$ & $13388,89 \mathrm{a}$ \\
AG 7391 & $15772,22 \mathrm{a}$ & $15761,11 \mathrm{a}$ & $15994,44 \mathrm{a}$ & $16072,22 \mathrm{a}$ & $14750,00 \mathrm{a}$ & $15211,11 \mathrm{a}$ \\
PIONEER 3232 & $14583,33 \mathrm{a}$ & $13294,44 \mathrm{a}$ & $11283,33 \mathrm{a}$ & $13650,00 \mathrm{a}$ & $13388,89 \mathrm{a}$ & $14566,67 \mathrm{a}$ \\
DINA 170 & $13472,22 \mathrm{~b}$ & $11838,89 \mathrm{~b}$ & $16527,78 \mathrm{a}$ & $16472,22 \mathrm{a}$ & $9894,44 \mathrm{~b}$ & $11222,22 \mathrm{~b}$ \\
AG 1051 & $15150,00 \mathrm{a}$ & $15222,22 \mathrm{a}$ & $16511,11 \mathrm{a}$ & $16433,33 \mathrm{a}$ & $14722,22 \mathrm{a}$ & $15294,44 \mathrm{a}$ \\
EMGOPA 501 & $10794,44 \mathrm{~b}$ & $11000,00 \mathrm{~b}$ & $15083,33 \mathrm{a}$ & $12038,89 \mathrm{~b}$ & $10088,89 \mathrm{~b}$ & $10394,44 \mathrm{~b}$ \\
AG 603 & $17133,33 \mathrm{a}$ & $13977,78 \mathrm{a}$ & $16522,22 \mathrm{a}$ & $15850,00 \mathrm{a}$ & $14127,78 \mathrm{a}$ & $14494,44 \mathrm{a}$ \\
BR 112 & $11611,11 \mathrm{a}$ & $13661,11 \mathrm{a}$ & $13844,44 \mathrm{a}$ & $14794,44 \mathrm{a}$ & $12872,22 \mathrm{a}$ & $11244,44 \mathrm{a}$ \\
BR 106 & $12561,11 \mathrm{a}$ & $12950,00 \mathrm{a}$ & $13383,33 \mathrm{a}$ & $11700,00 \mathrm{a}$ & $13288,89 \mathrm{a}$ & $13272,22 \mathrm{a}$ \\
BR 126 & $12850,00 \mathrm{~b}$ & $12666,67 \mathrm{~b}$ & $15844,44 \mathrm{a}$ & $16277,78 \mathrm{a}$ & $12383,33 \mathrm{~b}$ & $11466,67 \mathrm{~b}$ \\
CMS 39 & $12694,44 \mathrm{a}$ & $11783,33 \mathrm{a}$ & $11955,56 \mathrm{a}$ & $13733,33 \mathrm{a}$ & $12833,33 \mathrm{a}$ & $11055,56 \mathrm{a}$ \\
CMS 50 x 28 & $10300,00 \mathrm{a}$ & $10605,56 \mathrm{a}$ & $11466,67 \mathrm{a}$ & $11055.56 \mathrm{a}$ & $10433,33 \mathrm{a}$ & $12311,11 \mathrm{a}$ \\
\hline
\end{tabular}

${ }^{1}$ Médias seguidas da mesma letra, na linha, não diferem estatisticamente pelo teste de Scott-Knott a $\%$ de probabilidade.

de que quando se têm materiais com tipo de interferência diferenciada de cada lado da parcela, os arranjos 1 e 2 apresentam resultados semelhantes, mas quando os materiais apresentam o mesmo tipo de interferência de cada lado da parcela, os resultados obtidos por esses arranjos são diferentes.

Com relação ao arranjo 3 (Tabela 5), observa-se que $85 \%$ dos materiais estudados apresentaram informações semelhantes às dos arranjos 1 e 2 . A ausência de interação genótipo x posição obtida através da utilização do arranjo 4 (Tabela 1), indica que o efeito de bordadura sobre os genótipos avaliados foram semelhantes, porém mesmo neste caso deve-se levar em consideração o efeito significativo da posição, pois a menor produção das linhas externas poderia acarretar subestimativas de rendimentos caso elas fossem consideradas na análise do experimento.

Considerando que nos arranjos 1, 2 e 3,30\%, $20 \%$ e $25 \%$ respectivamente, dos materiais estudados poderiam ter sofrido influência da parcela adjacente, uma vez que o rendimento desses materiais foi afetado e que esta influência poderia prejudicar a comparação entre os tratamentos, recomenda-se, a partir dessas informações, o uso de duas fileiras de bordadura de cada lado da parcela experimental. Contudo, recomendam-se mais pesquisas a fim de determinar se uma fileira de bordadura lateral como empregado hoje pelos pesquisadores em seus ensaios não está levando a uma subestimação do rendimento de alguns genótipos.

Com base nessas informações, analisaram-se os dados da variável PEP, considerando a área útil e a área total da parcela. Observa-se, pela Tabela 6, que a comparação entre os tratamentos foi alterada, ficando os genótipos DINA 170, BR 126 e XL 655 prejudicados quando se consideraram todas as linhas da parcela na análise do experimento, pois esses materiais apresentaram redução, no rendimento, em $19,77 \%, 15,44 \%$ e $9,25 \%$ respectivamente. Esses resultados mostram que o uso de fileiras de bordadura lateral muitas vezes deve ser recomendado como forma de aumentar a precisão experimental e evitar recomendações inadequadas de genótipos. 
TABela 6. Peso médio das espigas com palha de 20 genótipos de milho-verde, com o uso ou não de 2 linhas de bordadura lateral ${ }^{1}$.

\begin{tabular}{lcrr}
\hline \multirow{2}{*}{ Genótipo } & \multicolumn{3}{c}{ Peso das espigas (kg/ha) } \\
\cline { 2 - 4 } & Área útil & Área total & \multicolumn{1}{c}{ Diferença } \\
\hline AG 951 & $18566,67 \mathrm{a}$ & $16887,04 \mathrm{a}$ & 1679,63 \\
DINA 170 & $16500,00 \mathrm{a}$ & $13237,96 \mathrm{~b}$ & 3262,04 \\
AG 1051 & $16472,22 \mathrm{a}$ & $1555,56 \mathrm{a}$ & 916,67 \\
AG 603 & $16186,11 \mathrm{a}$ & $15350,93 \mathrm{a}$ & 835,19 \\
BR 126 & $16061,11 \mathrm{a}$ & $13581,48 \mathrm{~b}$ & 2479,63 \\
AG 7391 & $16033,33 \mathrm{a}$ & $15593,52 \mathrm{a}$ & 439,81 \\
C 742 & $15488,89 \mathrm{a}$ & $14813,89 \mathrm{a}$ & 675,00 \\
XL 604 & $15505,56 \mathrm{a}$ & $15217,59 \mathrm{a}$ & 287,96 \\
XL 660 & $15166,67 \mathrm{a}$ & $14450,00 \mathrm{a}$ & 716,67 \\
XL 655 & $15102,78 \mathrm{a}$ & $13705,56 \mathrm{~b}$ & 1397,22 \\
AG 4591 & $14741,67 \mathrm{a}$ & $15153,70 \mathrm{a}$ & $-412,04$ \\
AG 519 & $14430,56 \mathrm{~b}$ & $13210,19 \mathrm{~b}$ & 1220,37 \\
BR 112 & $14319,44 \mathrm{~b}$ & $13004,63 \mathrm{~b}$ & 1314,81 \\
AGROMEN 2012 & $13961,11 \mathrm{~b}$ & $14019,44 \mathrm{~b}$ & $-58,33$ \\
EMGOPA 501 & $13561,11 \mathrm{~b}$ & $11566,67 \mathrm{c}$ & 1994,44 \\
C 505 & $13355,56 \mathrm{~b}$ & $13612,96 \mathrm{~b}$ & $-257,41$ \\
CMS 39 & $12844,44 \mathrm{~b}$ & $12342,59 \mathrm{c}$ & 501,85 \\
BR 106 & $12541,67 \mathrm{~b}$ & $12859,26 \mathrm{~b}$ & $-317,56$ \\
PIONEER 3232 & $12466,67 \mathrm{~b}$ & $13461,11 \mathrm{~b}$ & $-994,44$ \\
CMS 50 x 28 & $11261,11 \mathrm{~b}$ & $11028,70 \mathrm{c}$ & 232,41 \\
\hline
\end{tabular}

${ }^{1}$ Médias seguidas da mesma letra, na linha, não diferem estatisticamente pelo teste de Scott-Knott a 5\% de probabilidade.

\section{CONCLUSÕES}

1. Todos os arranjos permitem verificar que há necessidade de bordadura lateral

2. O arranjo 1, no qual o efeito de bordadura é estudado de forma que os resultados convirjam para o centro da parcela, e o arranjo 2, no qual este efeito é estudado de cada lado da parcela, são os que apresentam maior precisão nos resultados.

3. Em estudos com a cultura de milho-verde é necessário o uso de duas fileiras de bordadura lateral de cada lado da parcela.
4. A competição afeta a comparação entre os tratamentos.

\section{REFERÊNCIAS}

ARRUDA, H.V. Sobre a necessidade de fileiras de bordadura, em experiências de campo. Bragantia, Campinas, v.18, n.1, p.101-106, 1959.

GOMEZ, K.A. Border effects in rice experimental plots. II. Varietal compettion. Experimental Agriculture, Cambridge, Grã-Bretanha, v.8, p.295-298, 1972.

GOMEZ, K.A.; GOMEZ, A.A . Statistical procedures for agricultural research. New York : J. Willey \& Sons, 1984. 680p.

SCAPIM, C.A.; CARVALHO, C.G.P. de; CRUZ, C.D. Uma proposta de classificação dos coeficientes de variação para a cultura do milho. Pesquisa Agropecuária Brasileira, Brasília, v.30, n.5, p.683686, maio 1995.

SILVA, P.S.L.; SOUZA, P.G.; MONTENEGRO, E.E. Efeito de bordaduras nas extremidades de parcelas de milho irrigado. Revista Ceres, Viçosa, v.38, n.216, p.101-107, 1991.

VALENTINI, L.; VIEIRA, C.; CONDÉ, A.R.; CARDOSO, A.A. Fileiras de bordadura em ensaios de competição entre variedades de feijão. Ciência e Cultura, São Paulo, v.40, n.10, p.1004-1007, out. 1988.

VIEIRA, C. Melhoramento do feijoeiro (Phaseolus vulgaris L.) no Estado de Minas Gerais. I. Ensaios comparativos e de variedades realizados no período de 1956 a 1961. Experientiae, v.4, p.1-68, 1964.

ZIMMERMANN, F.J.P. Efeito de bordadura em parcelas experimentais de arroz de sequeiro. Pesquisa Agropecuária Brasileira, Brasília, v.15, n.3, p.297300, jul. 1980. 